\title{
INFLUENCE OF THE MAGNETIC FIELD ON THE VISCOSITY COEFFICIENT OF LUBRICOOLANT THAT IS USED IN THE CUTTING PROCESS
}

\author{
Umarov Erkin Odilovich ${ }^{1}$, Mardonov Umidjon Tokhir Ugli ${ }^{1}$, Shoazimova Umida Khasanovna ${ }^{1}$ \\ ${ }^{1}$ Tashkent State Technical University, Uzbekistan \\ Email: umid.mardonov@tdtu.uz
}

\begin{abstract}
Nowadays, a number of scientific studies have been conducted to study the properties of magnetic fields and their effects on various fluids. In this article, the authors summarize the results of their study on the effects of a magnetic field on several different flowing fluids and no flowing fluids. They experimented with liquids such as ordinary water, $\mathrm{K}_{2} \mathrm{Cr}_{2} \mathrm{O}_{7}$ (Chromic). During the study, they focused on the effect of a magnetic field at different inductions on the viscosity coefficient of the fluids in a sample. In this article, they give the results of the experiment which has conducted with water. The results of the study and the authors' conclusions are presented in the article.
\end{abstract}

Keywords: Cutting Process, Lubricoolant, Magnetic Field, Metal Cutting, Machining, Viscosity Coefficient.

\section{Introduction}

Many research works have been done on the effects of magnetic fields on the various physical and chemical properties of liquids. Especially in the last decade, a lot of work has been completed on this and a number of noticeable results have been obtained [1]. Han and others studied the optical properties of water between two strong magnets and found that the magnetic field changes the infrared light absorption of water [2]. Holysz determined that magnetic fields increase water permeability and reduce surface tension $[3,4]$. Wang with his team studied the effects of a static magnetic field on a fluid in friction experiments, and the results show that the coefficient of friction in a magnetic field is lower [5]. Moreover, V.E. Nikolsky studied the effects of magnetic and electromagnetic fields on the gas and liquid contact phases with analytical and experimental ways. M. Amiri and A.A. The Dadkhans [16] determined whether physical cleaning of water would result in a decrease in its surface tension. V.N. Tirtigin [13] and others studied the effect of a weak electromagnetic pulse field on the microflora of lubricating cooling liquid. The work about the effect of the magnetic field on the composition of water and the organisms in it (shrimp in saltwater, A. salina) was experimented by S.M. Hassan and R.A. Rahman [11].

However, studies about the comparison of the effects of magnetic fields on flowing and stationary fluids are insufficient. Nevertheless, there is no experimental study that shows the effect of magnetic fields on the viscosity coefficient of the liquids. Such properties of the liquid are very important in the field of mechanical engineering, especially which greatly helps to reduce the unpleasant odor of the lubricating coolant during the cutting process of the machine in order to cool the cutting environment. The purpose of this article is to analyze the effect of a magnetic field on the viscosity coefficient of several fluids at stationary and flowing conditions. In addition, experiments were performed on various inductions of the magnetic field and very useful results were obtained from them.

\section{Methods and Materials}

The use of special lubricating coolants in the cutting process - lubricating cooling liquid conditions increases the stability of the cutting tool, reduces cutting forces, improves the quality of the finished surface, increases the operational durability of the product, increases labor productivity, etc. The essence of the lubricating-cooling technological environment as a lubricant in the cutting process is to reduce the friction and adhesion processes by interacting with the tool, chip, cutting surface with friction surfaces. As a result, the degree of deformation and wear of the cutter is reduced, the cutting temperature is lowed, and the resulting surface roughness is reduced significantly. The cooling capacity of lubricating cooling liquid conditions is determined by their thermos-physical parameters: thermal conductivity, heat capacity, kinetic cohesion, and thermal spatial variation. 
Observations have shown that during the cutting process not only decrease the strength of contact layers of the cutting tool but also have conditions for hardening. For high-speed steel, this condition corresponds to a temperature of $200-300^{\circ} \mathrm{C}$ and a pressure of up to $500 \mathrm{MPa}$ (low level). The upper level is the resistance of the cutting blade to brittle wear. At this pressure limit, the tightening efficiency is higher than the relatively normal tension.

The washing effect is the continuous removal of the waste which was generated during the cutting process from the cutting area: the chip, small part of chips, the products after cutting, the ash, sand, and other deposits, the elements on the surface of the detail which were leftover from previous operations. In addition, ensuring that particles from grinding do not get into the grinding tool, Protecting the juvenile surfaces during the cutting process from the adverse effects of the atmosphere, corrosion protection, loss of strength, and performance characteristics of the part. Various chemically active substances are added to enhance the effect of lubricating cooling liquid on the cutting process.

It is known that the magnetic field has a sufficient effect on the properties of liquids, which means that the magnetic field must also affect the lubricating cooling liquid used in the cutting process[10]. As well as, that shows a significant effect on the cutting process. From this point of view, it is technologically and economically important to study the effect of the magnetic field on some of the properties of the lubricating cooling liquid used in the cutting process.

First of all, we examined the effect on the viscosity coefficient of ordinary water, which is commonly used in the cutting process [it is clear that it affects the shear strength during cutting].

\section{Experiment}

\section{- Magnetization}

Two types of fluids were used in the experiment: ordinary water, which is supplied by the local cold water supply, and lubricating coolant $\mathrm{K}_{2} \mathrm{Cr}_{2} \mathrm{O}_{7}$ (Chromic), which is used in the laboratory of the Department of Mechanical Engineering, Tashkent State Technical University.

A special laboratory device based on a solenoid was projected and built to magnetize the liquids (Figure 1).

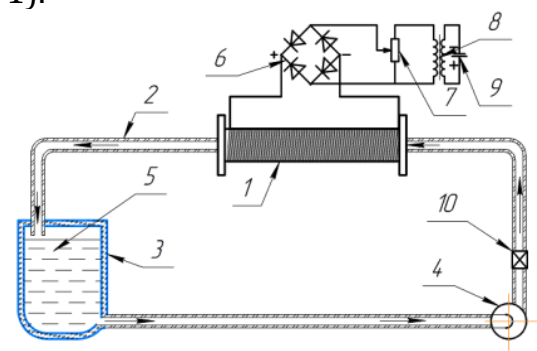

Figure 1: 1-Solenoid, 2-Ceramic pipe, 3-container, 4pump, 5-liquid, 6-limiting velocity diode, 7-rheostat, 8transformer, 9-Voltage.
Container 3 is filled with 10 liters of sampled liquid 5 , and the liquid on the container is removed from the container using a pump 4 through ceramic tubes 2. The liquid that moving through the pipes following the direction that is shown in the diagram passes through the center of the solenoid 1 with a diameter of $30 \mathrm{~mm}$ and is poured back into the previous container. The liquid coming through the tube passes through the center of the solenoid, which creates a magnetic field, and then turns into a magnetized liquid and is poured back into the container. The pressure of the liquid is controlled by the tap 10 and the required pressure is obtained. After a few minutes of the cycle, the liquid in the container becomes fully magnetized and the viscosity coefficient of the liquid in it is measured.

The solenoid created for the experiment was made by wrapping an insulated copper wire with a diameter of $1.06 \mathrm{~mm}$ in a plasma coil with a radius of $30 \mathrm{~mm}$, and the number of turns of wires in it was 1200. The $220 \mathrm{~V}$ voltage from the source (9) is reduced by transformer 8 and passed through a rheostat 7 in order to turn to a regulated voltage with the help of diode bridge 6 . The regulated voltage from the diode bridge is transmitted to the solenoid and a magnetic field is created. The voltage value is controlled using a rheostat and is set to the desired voltage.

\section{- Measurement of the viscosity coefficient}

When one layer of all real fluids moves relative to another, more or less friction occurs. The accelerating force acts on the slower moving layer by the faster-moving layer and, conversely, the slowing down force acts on the faster-moving layer by the slower moving layer. These forces are called internal friction forces, which are directed along the surface of the interacting fluid layer. The surface area $\nabla S$ of the layer we are examining is much larger, the internal friction force $f$ is much greater, and also depends on how fast $\mathrm{n}$ the flow velocities between these layers change. If assuming that two layers which distance of them are $\nabla Z$ (Fig. 2) flow with velocities respectively $\vartheta_{1}$ and $\vartheta_{2}$, the velocity difference is $\vartheta_{1}-\vartheta_{2}=\Delta v$.

The distance between the layers is taken in the direction which is perpendicular to the flow rate. The magnitude of the change in velocity, which moves from one layer to another, is called the velocity gradient.

The internal friction force $\mathrm{f}$ is proportional to the velocity gradient $\Delta \vartheta / \Delta Z$ b and the friction surface $\mathrm{S}$.

$$
\Delta f=\eta \frac{\nabla \vartheta}{\nabla Z} \nabla S
$$


The magnitude $\eta$ that depends on the properties of the liquid is called the internal friction coefficient or viscosity coefficient of the liquid. If take from formula (1) $\frac{\nabla \vartheta}{\nabla Z}=1$ and $\mathrm{S}=1$, we get $\mathrm{r}=\mathrm{f}$. This means that the coefficient of adhesion is numerically equal to the frictional force which a velocity gradient is 1 and prepared between the layers on a unit surface. The viscosity coefficient depends on the properties and temperature of the liquid. As the temperature rises, the viscosity decreases. The unit of viscosity in the SGS system is Pz (puaz), which is the coefficient of internal friction acting on a $1 \mathrm{sm}^{2}$ surface. To follow the size of viscosity (1) will be:

$$
[\eta]=-\left[\frac{f}{\frac{\Delta \vartheta}{\Delta Z} \cdot \Delta S}\right]=\mathrm{L}^{-1} \mathrm{MT}^{-1}
$$

The water viscosity which depends on the temperature is as follows:

in $0^{\circ} \mathrm{S} \eta=0.1775$ puaz, in $20,5^{\circ} \mathrm{S}, \eta^{=0.01}$

If an object moves in a non-sticky liquid, the liquid will not resist its movement. Resistance occurs only when the body moves in a viscous environment. The fluid follows the body and moves more slowly. As a result, frictional forces are created between the layers of fluid.

In this experiment, steel or lead balls with a diameter of about 1-2 mm are used as a solid. These spherical balls are thrown into the liquid one by one. If all the sides of a ball are in contact with a liquid (without gas bubbles around it) and fall behind it at a small speed without forming a vortex, the gravitational force which is exerted by the liquid is found according to Stokes' law.

$$
f=6 \pi \cdot \eta \cdot \vartheta \cdot r
$$

Where,

$$
\begin{aligned}
& \eta \text { - Coefficient of viscosity or internal friction } \\
& \vartheta \text { - The falling velocity of the ball } \\
& \mathrm{r} \text { - The radius of the ball }
\end{aligned}
$$

Three forces impact on a ball moving in a viscous liquid; (1) gravitational force $\mathrm{P}$, the lifting force of a liquid according to Archimedes' law $\vec{f}$, the force of internal friction $\vec{f}_{2}$. These three forces are directed in a straight line (gravity downwards, fluid lifting force, and resistance upwards). As the falling velocity of the ball decreases (in proportion to it), the resistance of the fluid also increases.
When the falling velocity of the ball reaches a certain value $\vartheta_{0}$, the force acting on it is equal to zero (the sum of the upward resistance force and the Archimedean force is equal to the gravitational force).

$$
R=f_{1}-f_{2}=0
$$

However, the ball continues to move because of its energy (Newton's first law). Due to the size $\frac{4}{3} \pi r^{3}$

$\frac{4}{3} \pi r^{3}$ of the ball, its weight is as follows:

$$
P=m g=\rho v g=\frac{4}{3} \pi r^{2} \rho g
$$

Because of the squeezed volume of liquid is equal to the volume of the bubble lifting(Archimedes) force;

$$
f_{\gamma}=\frac{4}{3} \pi \cdot r^{3} \rho_{0} g
$$

The friction force is based on (3);

$$
f_{2}=6 \pi \eta \cdot r \ell_{0} r
$$

Substituting (5) (6) and (7) into (4) gives the following equation;

$$
\frac{4}{3} \pi \cdot r^{3}\left(\rho-\rho_{0}\right) g=6 \pi \cdot r \vartheta_{0} \cdot \eta
$$

Where,

$\rho$ - the density of the ball

$\rho_{0}$ - the density of the liquid

$\mathrm{g}$ - acceleration of free fall

If the ball passes $h$ height with $t$ time in a liquid $\vartheta_{0}=\frac{h}{t}$

Putting it in (8), we get what

$$
\eta=\frac{2\left(\rho-\rho_{0}\right)}{9 h} g \cdot r^{2} t
$$

All values on the right side of the equation are determined during the experiment, accordingly from that we can find the coefficient of internal friction of the fluid.

\section{- Measurement of the viscosity coefficient}

The instrument consists of a glass cylinder device, which is fallen with the testing liquids (glycerin, cottonseed oil, and water). The cylinder has two height indicators at a distance $\mathrm{h}$ from each other. 


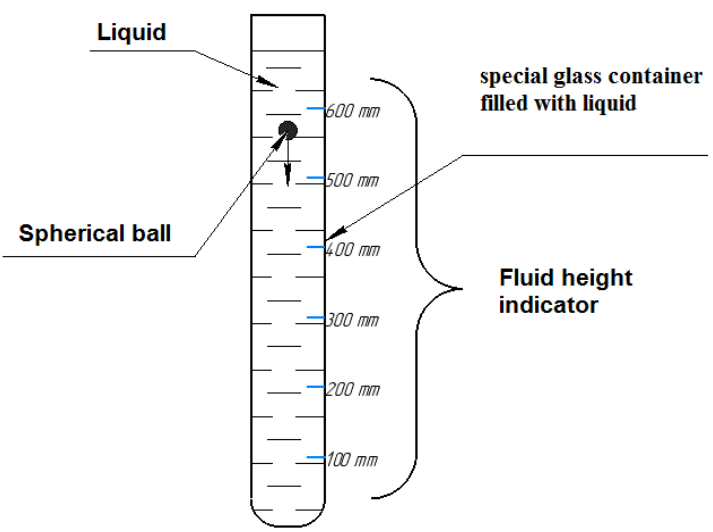

Figure 2. A device for measuring the coefficient of internal friction of a fluid according to Stock's method

The diameter of the ball is measured to the accuracy at $0.01 \mathrm{~mm}$ using a micrometer. The ball is thrown into the liquid inside the cylinder (the ball should be thrown as close to the cylinder axis and the surface of the liquid as possible). The stopwatch is started as the ball passes through the $\mathrm{d}_{1}$ mark on the cylinder and stops as it passes over the $\mathrm{d}_{2}$ mark. That measures the time taken for the ball to pass the distance $h$ from $d_{1}$ to $d_{2}$ (Figure 3 ).

Measurement of the distance between $d_{1}$ and $d_{2}$ is found by using the ruler. The experiment is performed with at least 10 balls for each liquid. Knowing the values of $\kappa, h, \rho, \rho_{0}$ and $\mathrm{t}$, the viscosity coefficient of the fluid is determined using the formula (8), $\rho$ and $\rho_{0}$ are taken from the table.

The reached result is recorded in table 1.

\section{Results and Discussions}

\section{- Viscosity coefficient of tap water}

We initially performed the experiment in ordinary water. For this, we took a sample from the local tap water and determined the viscosity coefficient of the sample obtained by the Stokes method. To do this, we poured water at a temperature of $20^{\circ} \mathrm{C}$ into a cylindrical flask with a diameter of $40 \mathrm{~mm}$ at a height of $570 \mathrm{~mm}$ and determined the coefficient of internal friction of the water in the sample using steel balls with a diameter of $4.3 \mathrm{~mm}$ and a density of $7800 \mathrm{~kg} / \mathrm{m}^{3}$. We performed the experiment 5 times and the obtained results are given in Table 1 below.

Table 1. Ultimate tensile strength values and elongation to fracture

\begin{tabular}{|c|c|c|c|c|c|c|c|c|}
\hline № & $\begin{array}{c}\mathrm{r}, \\
\mathrm{mm}\end{array}$ & $\begin{array}{c}\mathrm{h}, \\
\mathrm{mm}\end{array}$ & $\mathrm{t}, \mathrm{s}$ & $\eta$ & $\eta_{\mathrm{a}}$ & $\Delta \eta$ & $\Delta \eta_{\mathrm{a}}$ & $\varepsilon, \%$ \\
\hline 1 & 2.15 & 570 & 0.74 & 0.088 & 0.088 & 0 & 0.0032 & 3.6 \\
\hline 2 & 2.15 & 570 & 0.79 & 0.094 & 0.088 & 0.006 & 0.0032 & 3.6 \\
\hline 3 & 2.15 & 570 & 0.71 & 0.085 & 0.088 & 0.003 & 0.0032 & 3.6 \\
\hline 4 & 2.15 & 570 & 0.70 & 0.084 & 0.088 & 0.004 & 0.0032 & 3.6 \\
\hline 5 & 2.15 & 570 & 0.76 & 0.091 & 0.088 & 0.003 & 0.0032 & 3.6 \\
\hline
\end{tabular}

Using the indication of our firs experiment we built the following diagram:

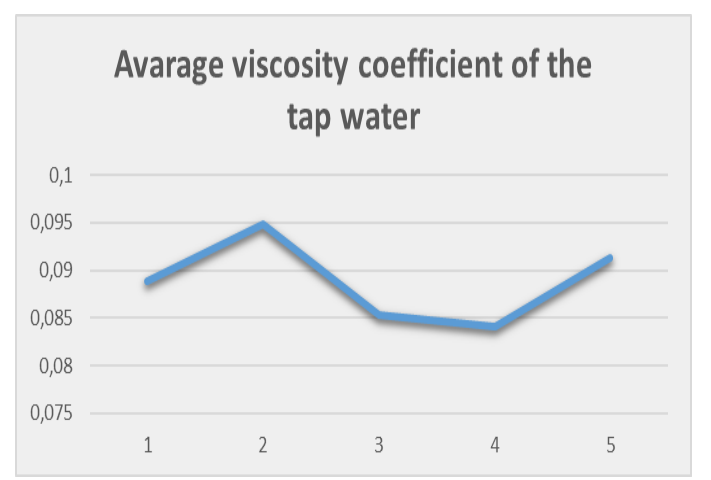

Figure 3: Average viscosity coefficient of tap water after 5 times experiment 
- Effect of magnetic field on viscosity coefficient flowing water

In Experiment 2, we used water that had the same physical and chemical properties as the water used in the first. For the sample, we poured 10 liters of water into a container in the laboratory device shown in Figure 1 to magnetize it. With the help of the pump, the water coming out of the container is magnetized and poured back into the container. The current transmitted to the transformer from a source connected to the $220 \mathrm{~V}$ voltage is reduced to $40 \mathrm{~V}$ voltage by a step-down transformer. The $40 \mathrm{~V}$ current coming out from the transformer is converted to alternating current by a diode bridge and transmitted to the solenoid. A magnetic field is generated along the central axis of a solenoid connected to a constant voltage of $40 \mathrm{~V}$, and the moving water passes through the magnetic field generated at the center of the solenoid and is converted into magnetized water. We conducted the magnetization process for 30 minutes and magnetized the moving water. Using the Stokes method performed in Experiment 1, taking a sample of the water in the container, we determined its viscosity coefficient. The results are presented in Table 2 below:

Table 2. Results taken from the measuring of the magnetized water viscosity coefficient

\begin{tabular}{|c|c|c|c|c|c|c|c|c|}
\hline № & $\begin{array}{c}\mathrm{r}, \\
\mathrm{mm}\end{array}$ & $\begin{array}{c}\mathrm{h}, \\
\mathrm{mm}\end{array}$ & $\mathrm{t}, \mathrm{s}$ & $\eta$ & $\eta_{\mathrm{a}}$ & $\Delta \eta$ & $\Delta \eta_{\mathrm{a}}$ & $\varepsilon, \%$ \\
\hline 1 & 2.15 & 570 & 0.65 & 0.078 & 0.079 & 0.001 & 0.002 & 2.5 \\
\hline 2 & 2.15 & 570 & 0.69 & 0.082 & 0.079 & 0.003 & 0.002 & 2.5 \\
\hline 3 & 2.15 & 570 & 0.64 & 0.076 & 0.079 & 0.003 & 0.002 & 2.5 \\
\hline 4 & 2.15 & 570 & 0.67 & 0.080 & 0.079 & 0.001 & 0.002 & 2.5 \\
\hline 5 & 2.15 & 570 & 0.68 & 0.081 & 0.079 & 0.002 & 0.002 & 2.5 \\
\hline
\end{tabular}

Comparing the results of the above experiments, we have created the following figure 4:

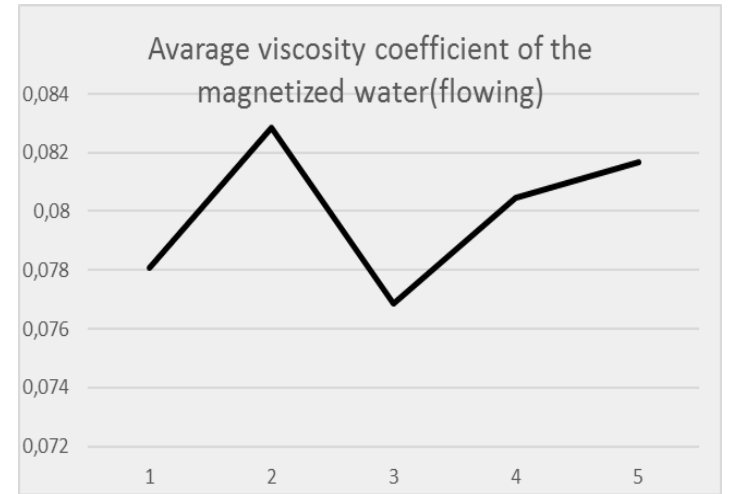

Figure 4. Average viscosity coefficient of tap water after 5 times experiment

It is clear from the results which are obtained from two experiments above that the magnetic field influence on the viscosity coefficient of the water. Obtained results show that the viscosity coefficient of water decreases when the magnetic field effects on it. To clarify our research results, we make the line graph that accurately compares the difference of viscosity coefficient between magnetized and not magnetized water (Fig.5).

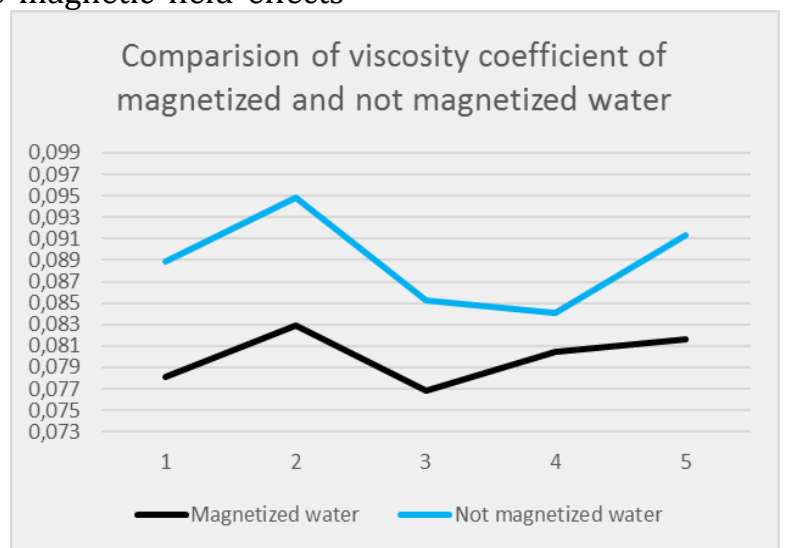

Figure 5. Difference between viscosity coefficient of magnetized and not magnetized water 


\section{Conclusions}

Based on the results, it can be said that the magnetic field causes a decrease in the viscosity coefficient of the moving fluid. The effect of the magnetic field on this property of liquids can be applied in many fields of mechanical engineering, especially in lubricating coolants in the cutting process. The application of this process can be a new step in the field of mechanical engineering.

From the experiments above, it can be seen that the properties of lubricating coolants used in the cutting process can be controlled by the magnetic field influencing it. This means it is possible to increase the durability of the cutting tool, improve the quality of the working surface, and reduce the product recognition price (planned for future implementation). In addition, the environmental issue in production will be improved.

Not at the expense of chemically active substances, but at the expense of an environmentally friendly magnetic field (also economically beneficial). It is well known that lubricants start to emit unpleasant odors over time[9] and we get rid of it.

\section{Acknowledgements}

The authors would like to acknowledge Professors of the "Physics" department of Tashkent State Technical University, Ministry of Higher Education Uzbekistan, and mechanics of the "Mechanical engineering" department.

\section{References}

[1] Prof. E.O. Umarov, Kh.Z.Abduraxmonov, U.T. Mardonov, D.R. Dustmurodov. Studying the influence of magnetic and electromagnetic field on fluids. https://www.ijiert.org/paperdetails.php?paper id=141747

[2] Han X, Peng Y, Ma Z. Effect of magnetic field on optical features of water and $\mathrm{KCl}$ solutions. Optik-Int J Light Electron Optics 2016;127(16):6371-6. https://
[3] Holysz L, Szczes A, Chibowski E. Effects of a static magnetic field on water and electrolyte solutions. J Colloid Interface Sci 2007;316(2):996.

https://doi.org/10.1016/j.jcis.2007.08.026.

[4] Amiri MC, Dadkhah AA. On reduction in the surface tension of water due to magnetic treatment. Colloids Surf A 2006;278(1):252-5. https://doi.org/10.1016/j.colsurfa.2005.12.046

[5] Wang Y, Zhang B, Gong Z, et al. The effect of a static magnetic field on the hydrogen bonding in water using frictional experiments. J Mol Struct 2013;1052(11):102-4.

https://doi.org/10.1016/j.molstruc. 2013.08.021.

[6] M. C. Amiri and Ali A. Dadkhah, "On Reduction in the Surface Tension of Water Due to Magnetic Treatment," Colloids and Surfaces A: Physicochemical and Engineering Aspects 278, no. 1-3 (2006): 252-55, https://doi.org/10.1016/j.colsurfa.2005.12.046

[7] I.G. Shayxiev et al., "UDC 66.086.4," n.d.,"Vliyanie Elektromagnitnogo impulsnogo polya na mikrofloru smazochno-oxlajdayuщеy jidkosti[Influence of the Electromagnetic Pulse Field on the microflora of the cutting fluid]" n.d.

[8] S. M. Hassan and Ridzwan Abdul Rahman, "Effects of exposure to magnetic field on water properties and hatchability of Artemia Salina" 11, no. 11 (2016): 416-23, www.arpnjournals.com.

[9] T.U. Umarov, U.T. Mardonov, O.A. Khasanov, Sh.O. Ozodova, B.D. Yusupov. Research on the variation of firmness of pointed drills by the method of simulation modeling of the process of wear. https://www.psychosocial.com/article/PR201 298/12155/.

[10] Cao, J., Wang, F., Guo, K., Kassim, S. Design and electromagnetic thermal analysis of electric vehicle hub motor based on finite element simulation model (2019). International Journal of Mechatronics and Applied Mechanics, 2 (6), pp. 151-156. 